\title{
Adding Adaptability to Mailbox-Based Mobile IP
}

\author{
Liang Zhang ${ }^{1}$, Beihong $\mathrm{Jin}^{1}$, and Jiannong $\mathrm{Cao}^{2}$ \\ ${ }^{1}$ Institute of Software, Chinese Academy of Sciences, \\ Hai Dian, Beijing, PRC \\ \{zhangliang1216,jbh\} @otcaix.iscas.ac.cn \\ ${ }^{2}$ The Hong Kong Polytechnic University, \\ Hung Hom, Kowloon, Hong Kong \\ csjcao@comp.polyu.edu.hk
}

\begin{abstract}
Mobile IP is one of the dominating protocols that provide mobility support in the Internet. However, even with some proposed optimization techniques, there is still space for improving the performance. In this paper, we present a mailbox-based scheme to further improve the performance. In this scheme, each mobile node migrating to a foreign network is associated with a mailbox. A sender sends packets to the receiver's mailbox, which will in turn forward them to the receiver. During each handoff, a mobile node can decide whether to move its mailbox and report the handoff to the home agent, or simply to report the handoff to the mailbox. In this way, both the workload on the home agent and the registration delay can be reduced. Also, the scheme is adaptive. By applying the dynamic programming to compute the optimal mailbox's migration policy, the scheme can make the total cost minimized.
\end{abstract}

Keywords: mobile computing, mailbox, mobile IP, dynamic programming.

\section{Introduction}

The growth of wireless communication technologies and the advancement of laptop and notebook computers induce a tremendous demand for mobile and nomadic computing. Researchers have investigated Internet Protocol (IP) for mobile internetworking, leading to the development of a proposed standard for IP mobility support called Mobile IP [1].

However, Mobile IP suffers from the well-known triangle routing problem where packages have to first take the detour to the home network before being forwarded to the foreign network where the mobile node is currently residing. To deal with this problem, Mobile IP route optimization [1] is proposed. Any node that communicates with a mobile node maintains a binding cache. When the home agent intercepts a packet for the mobile node outside the home network, it will send a binding update message to the sender, informing it of the mobile node's current care-of address. The sender then updates its binding cache and tunnels any ensuing packets for the mobile node directly to its care-of address. An extension to the registration process called smooth handoff [1] enables a foreign agent to also make use of binding update to reduce the packet loss during a handoff. The mobile node may request the new 
foreign agent to send to the previous foreign agent a binding update message called "Previous Foreign Agent Notification", which will enable the previous foreign agent to re-tunnel any packet for the mobile node to the new care-of address. Although Mobile IP and route optimization provide general mechanisms for mobility support in the Internet, there are still several performance problems that need to be addressed [6].

In this paper, we present a mailbox-based scheme to alleviate the performance problems stated above. Each mobile node migrating to a foreign network is associated with a mailbox. A sender sends packets to the receiver's mailbox, which will in turn forward them to the receiver. During each handoff, a mobile node can decide whether to move its mailbox and report the handoff to the home agent, or simply to report the handoff to the mailbox. In this way, both the workload on the home agent and the registration delay can be reduced. Since the mailbox is located somewhere in the network closer to the receiver than the sender, the retransmission cost for the lost packets can also be reduced. By separating the mailbox from its owner, we can achieve adaptive location management that enables the dynamic tradeoff between the packet delivery cost and the location registration cost to minimize the total cost.

The rest of the paper is organized as follows. Section 2 presents our mailbox-based scheme. Section 3 proposes an adaptive algorithm to optimize the performance of our scheme. Section 4 evaluates the performance. The final section concludes the paper.

\section{A Mailbox-Based Scheme}

In this paper, both home agents and foreign agents are referred to as mobility agents. Each mobile node is associated with a mailbox, which is a data structure residing at a mobility agent. As shown in Fig. 1, if a sender wants to send a packet to a mobile node, it will simply send the packet to the receiver's mailbox. Later, the receiver receives the packet from its mailbox.

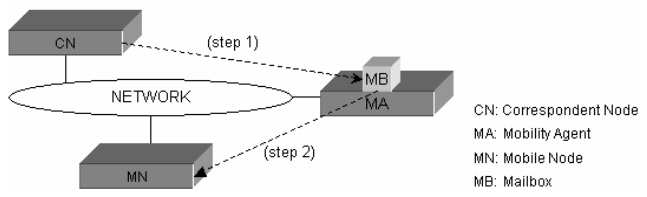

Fig. 1. Mailbox-based scheme

Initially, the mailbox is residing in the same network as its owner. The mobile node realizes that it has entered a new foreign network whenever it receives an "Agent Advertisement" message [1] from a new foreign agent. Immediately, it sends a registration message to the old foreign agent where its mailbox resides. The old foreign agent then decides whether or not to move the mailbox to the new foreign agent with the consideration of two primary factors: the distance to the new foreign agent and the communication traffic of the mobile node. If the mobile node is expected to receive many packets while the distance is long, it will be costly to forward all these packets to the new address and better to move the mailbox closer to the mobile node so as to 
achieve a more optimal route. On the other hand, if the mobile node seldom receives packets or the distance is quite short, it is economical to leave the mailbox at where it is to reduce the registration overhead. Therefore, how to decide the mailbox's migration pattern adaptively according to the two factors can affect greatly the performance of our scheme. We would like to postpone the discussion about this question in the next section. As a summary, we differentiate two types of handoff in our scheme, i.e., handoff without mailbox and handoff with mailbox, and we name them local handoff and home handoff, respectively.

Besides mailbox, another new data structure called address table is defined in each mobility agent. Each entry in an address table has six attributes: 1) the home address of the mobile node, 2) the mailbox's address, 3) a valid tag, 4) a pointer to the mailbox, 5) the care-of address of the mobile node, and 6) a time-to-live (TTL) timer. The valid tag is used to indicate whether the mailbox is under migration or not, which also implies whether the mailbox's address is outdated or not. The TTL timer is used to timeout trash entries in the address table. The scheme also defines operations for two processes, Migrating and Packet-forwarding.

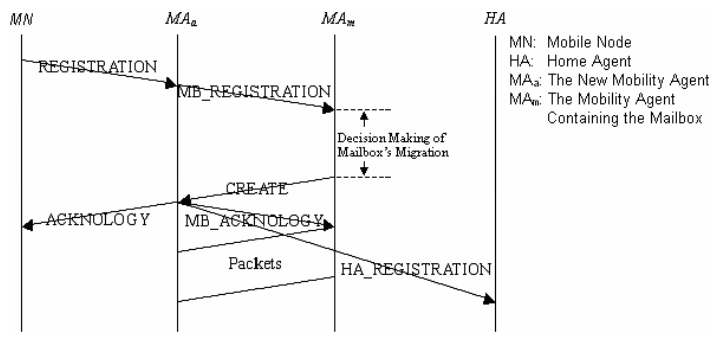

Fig. 2. Migrating

Upon receiving the advertisement from a new mobility agent $M A_{a}$, the mobile node $M N$ determines that it has roamed to a new foreign network. It then initiates the registration process as shown in Fig. 2. It first uses gratuitous ARP [2] to update the ARP caches of the nodes in the foreign network so that they will associate $M N$ 's link layer address with its home address. After that, it sends a "REGISTRATION" message to $M A_{a}$, within which the key information contained is the address of the mobility agent $M A_{m}$ where the mailbox $M B$ is currently residing.

Upon receiving the "REGISTRATION" message, $M A_{a}$ extracts the address of $M A_{m}$ from the message, and sends a "MB_REGISTRATION" message to $M A_{m}$.

Upon receiving the "MB_REGISTRATION" message, $M A_{m}$ makes a decision whether or not to move $M B$ to $M A_{a}$. In case that $M B$ does not migrate, $M A_{m}$ simply updates the care-of address of $M N$ to $M A_{a}$. Otherwise, it will:

- set the valid tag of the corresponding entry in the address table to false, and

- send a "CREATE" message to $M A_{a}$, requesting for a new mailbox $M B$ ' for $M N$.

Upon receiving the "CREATE" message, $M A_{a}$ creates $M B$ ' and adds an entry to its address table to record this newly created mailbox. It also sends three messages:

- an "ACKNOLOGY" message to $M N$, informing it of the new address of $M B$,

- an "MB_ACKNOLOGY" message to $M A_{m}$, telling it the creation of $M B$ ', and 
- an "HA_REGISTRATION" message to the home agent $H A$, registering the new address of the mailbox.

After receiving the "MB_ACKNOLOGY" message, $M A_{m}$ will:

- update the address of the mailbox in the address table to that of $M B$,

- set the valid tag to true,

- activate the TTL timer,

- stream every packet buffered in $M B$ to $M B$,

- inform the new address of the mailbox to the senders of the buffered packets in $M B$ by sending "UPDATE" messages, and

- after all the buffered packets have been streamed out, deconstruct the mailbox and setting null to these two attributes in the address table - the pointer to the mailbox and the care-of address of the mobile node.

After receiving the "HA_REGISTRATION" message, $H A$ updates the address of the mailbox in its address table.

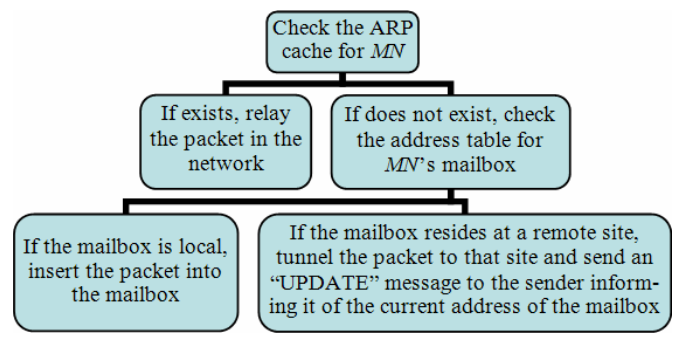

Fig. 3. Packet-forwarding

If a correspondent node $C N$ wants to send a packet to $M N$, it will first check its binding cache to see whether the address of $M N$ 's mailbox has been cached locally or not. If so, it will tunnel the packet to the cached address. Otherwise, it will send the packet with regular IP routing to $M N$ 's home address. Once the packet arrives in the home network, $H A$ will intercept the packet as it acts as a proxy ARP server for $M N$.

When a mobility agent receives a packet destined to $M N$, it will perform actions according to Fig. 3. For a packet in $M B$ to be forwarded to $M N, M A_{m}$ first checks the valid tag. If it is false, i.e., the mailbox is migrating to a new foreign agent, $M A_{m}$ will suspend the packet forwarding. It will rely on the migrating process to stream the packet to the new location of the mailbox later. Otherwise, the valid tag is true and $M A_{m}$ will directly tunnel the packet to the care-of address of $M N$.

\section{An Adaptive Algorithm}

In this section, we will first present the system model for a mobile network and the walk model for a mobile node, which are adopted in many existing studies such as [3] and [4]. The model assumes that the coverage area of a mobile network is partitioned into cells. A cell is defined as the coverage area of a mobility agent that can exchange packets with mobile nodes directly. One mobility agent serves only one cell and cells 
do not overlap with each other. A movement occurs when a mobile node moves from the residing cell to one of its neighboring cells. The distance between any two cells in the network is measured by the minimum number of cell boundary crossings for a mobile node to travel from one cell to another. If we assume that a mobility agent is a router in a cell that can communicate directly through wired lines with other mobility agents in the neighboring cells, the distance between two mobility agents can be defined as the distance between their cells.

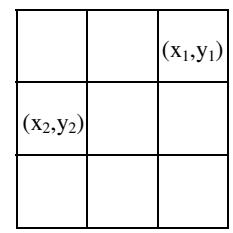

Fig. 4. System model

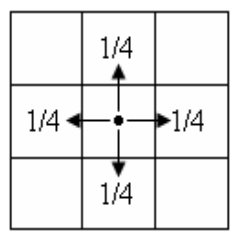

Fig. 5. Walk model

We consider a grid configuration for a mobile network, which is composed of equal-sized, rectangular and non-overlapping cells. With this configuration as shown in Fig. 4, each cell has four neighbors and the distance between any two cells with the coordinates $\left(\mathrm{x}_{1}, \mathrm{y}_{1}\right)$ and $\left(\mathrm{x}_{2}, \mathrm{y}_{2}\right)$ is $\left|\mathrm{x}_{2}-\mathrm{x}_{1}\right|+\left|\mathrm{y}_{2}-\mathrm{y}_{1}\right|$. We also consider a commonly used walk model - the random walk model for a mobile node. In this model, a mobile node moves to one of its four neighbors with equal probability of 1/4 as shown in Fig. 5.

Based on the model, we will develop an adaptive algorithm that can dynamically adjust the mailbox's migration pattern according to the two factors in order to optimize the performance. The performance metric is the total communication cost over the experimental period which includes both the location registration cost and the packet forwarding cost. The communication cost is defined as the multiplication of the number of messages sent, the message size and the traveling distance.

Table 1. Definition of parameters

\begin{tabular}{cl}
\hline Parameter & \multicolumn{1}{c}{ Definition } \\
\hline$f_{m}(t)$ & the (negative exponential) probability distribution function of the packet's inter-arrival time \\
$\lambda$ & the mean packet arrival rate, i.e., $f_{m}(t)=\lambda e^{-\lambda t}$ \\
$f_{r}(t)$ & the (negative exponential) probability distribution function of the mobile node's residence time at a cell \\
$\mu$ & the mean residence time at a cell, i.e., $f_{r}(t)=\mu e^{-\mu t}$ \\
$\eta$ & the expected number of packets to be received at a cell, which is known as the packet-to-mobility ratio \\
$\mathrm{d}_{\mathrm{Cs}}$ & $\begin{array}{l}\lambda / \mu ; \text { it is actually the second primary factor that may affect the mailbox's migration } \\
\text { the proportionality constant between the signaling transmission cost and the transmission distance }\end{array}$ \\
$\mathrm{d}_{\mathrm{Cp}}$ & the proportionality constant between the packet delivery cost and the transmission distance \\
$\mathrm{d}_{\mathrm{Ts}}$ & $\begin{array}{l}\text { the proportionality constant between the signaling transmission time and the transmission distance } \\
w\end{array}$ \\
$M$ & the proportionality constant between the transmission cost (the transmission time) of the wireless link \\
& and the number of correspondent nodes
\end{tabular}

In order to optimize the performance, we choose to use the dynamic programming since it can help to make a sequence of inter-related choices to optimize the system performance. Readers are referred to [5] for the detailed description and procedure 
about the dynamic programming. Before applying the dynamic programming, let us first precisely define our performance optimization problem. The experimental period starts with the mobile node and its mailbox collocating at an initial foreign agent, and ends after the mobile node has performed $N$ migrations. The total communication cost over this period can be expressed as follows.

$$
\operatorname{Cost}_{\text {total }}=\sum_{i=1}^{N}\left(\operatorname{Cost}_{\text {signaling }}(i)+\operatorname{Cost}_{\text {packet }}(i)\right)
$$

where $\operatorname{Cost}_{\text {signaling }}(i)$ and $\operatorname{Cost}_{\text {packet }}(i)$ means the location registration cost during the mobile node's $i$ th migration and the packet forwarding cost during the mobile node's residing at the new cell after its $i$ th migration, respectively. Our performance optimization problem is to minimize (1).

Fig. 6 depicts the network scenario about the mobile node's $i$ th migration in our scheme. The Current FA is the foreign agent after the mobile node's $i$ th migration from the Previous FA. Therefore, the two foreign agents must be adjacent to each other, i.e., $d_{5}=d \pm 1$ where $\boldsymbol{d}$ is actually the first primary factor that may affect the mailbox's migration and the \pm sign depends on whether the mobile node migrates away from or close to the mailbox. In case of home handoff, the Current FA will become the new residing place for the mailbox. Here $d_{2}, d_{4}$ and $d_{7}$ are the average distance to all the correspondent nodes.

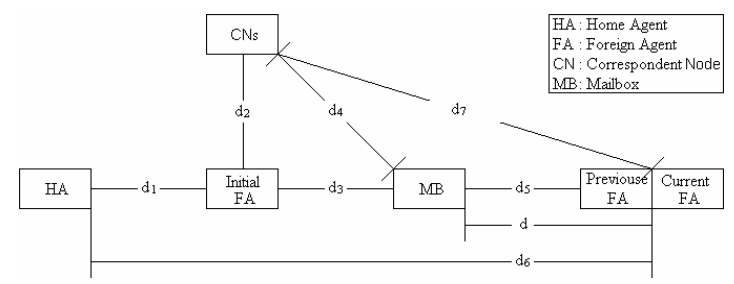

Fig. 6. Network scenario in our scheme

Depending on whether the $i$ th migration is a home handoff or a local handoff, Cost $_{\text {signaling }}(i)$ can be expressed as follows. Readers are referred to Fig. 2 for details.

$$
\operatorname{Cost}_{\text {signaling }}(i)= \begin{cases}\left(2 w+3 d+d_{6}\right) d_{C s}+\text { Cost }_{\text {update }}(i), & \text { a home handoff } \\ (w+d) d_{C s}, & \text { a local handoff }\end{cases}
$$

where $\operatorname{Cost}_{\text {update }}(i)$ means the signaling cost for the binding updates to the correspondent nodes during the mobile node's $i$ th migration and can be expressed as follows.

$$
\operatorname{Cost}_{\text {update }}(\mathrm{i})=\mathrm{M} \times \mathrm{d}_{4} \times \mathrm{d}_{\mathrm{Cs}}
$$

Now come to discuss the expression of $\operatorname{Cost}_{\text {packet }}(i)$. Normally, the packet forwarding cost, depending on the handoff type, can be expressed as follows.

$$
\text { Cost }_{\text {normal }}(i)= \begin{cases}\left(d_{7}+w\right) d_{C_{p}}, & \text { a home handoff } \\ \left(d_{4}+d+w\right) d_{C_{p}}, & \text { a local handoff }\end{cases}
$$


However, as packet loss may occur during the mobile node's migration, we have to also consider the packet retransmission cost for those lost packets. In our scheme, the lost packets are retransmitted from the nearby mailbox instead of the possibly far away senders. Therefore, the cost for successfully delivering any lost packet to the mobile node can be expressed as follows. Readers are referred to Fig. 6 for details.

$$
\text { Cost }_{\text {retransmis sion }}(i)=\left(d_{4}+d_{5}+w+d+w\right) d_{C p}
$$

During the period when the mobile node resides at the new cell after its $i$ th migration, it is expected to receive $\eta$ packets, within which a portion are retransmitted packets since they were delivered based on the outdated location information. As we know, the mailbox learns the new address of the mobile node only when it receives the "MB_REGISTRATION" message from the new foreign agent $M A_{a}$. Therefore, the period of the outdated location information can be expressed as follows.

$$
\mathrm{T}_{\text {retransmission }}(\mathrm{i})=(\mathrm{w}+\mathrm{d}) \mathrm{d}_{\mathrm{Ts}}
$$

As the mean packet arrival rate is $\lambda$, the mean lost packets is $\lambda \times T_{\text {retransmission }}(i)$. The mean packets that do not require retransmission are therefore $\eta-\lambda \times T_{\text {retransmission }}(i)$. Cost $_{\text {packet }}(i)$ can now be expressed as follows.

$$
\begin{aligned}
\operatorname{Cost}_{\text {packet }}(\mathrm{i})= & \left(\eta-\lambda \times \mathrm{T}_{\text {retransmission }}(\mathrm{i})\right) \times \operatorname{Cost}_{\text {normal }}(\mathrm{i})+ \\
& \lambda \times \mathrm{T}_{\text {retransmission }}(\mathrm{i}) \times \operatorname{Cost}_{\text {retransmission }}(\mathrm{i})
\end{aligned}
$$

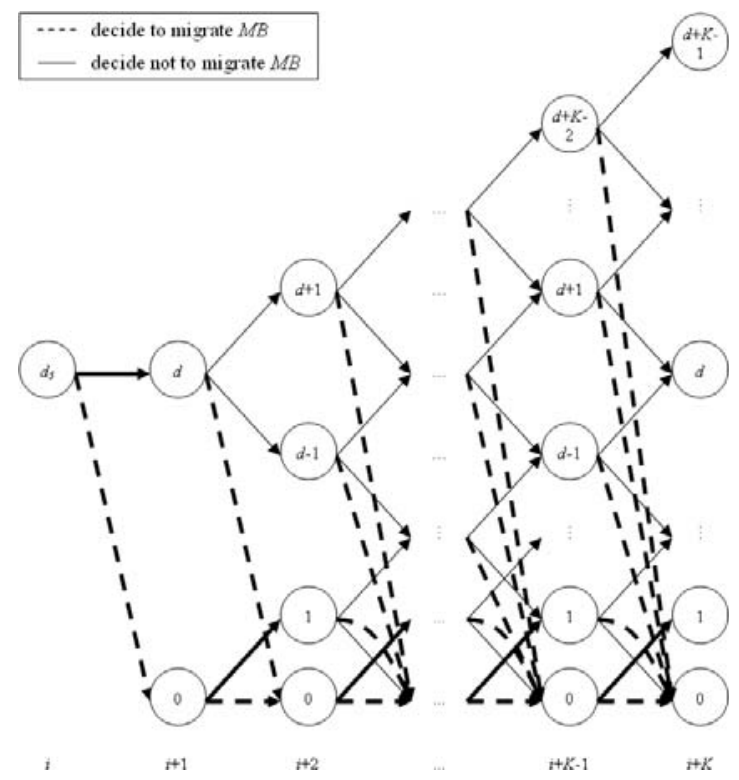

Fig. 7. Model for our cost optimization problem

Now let us start to formulate our cost optimization problem with the dynamic programming. Fig. 7 graphically depicts the model where the dynamic programming is utilized to optimize the communication cost for the next $K$ migrations of the mobile 
node. The circle represents the state, which means the distance between the mobile node and its mailbox before the current migration; and the arrow represents the action, which can be either a home handoff or a local handoff. Initially, the distance between the mobile node and its mailbox is $d_{5}$ according to Fig. 6 . The mobile node now starts its $i$ th migration. If this migration results in a home handoff, the system will go to state 0 since the mobile node and its mailbox are about to collocate. Otherwise, the system goes to state $d$ according to Fig. 6. Similarly, during the mobile node's $(i+1)$ th migration, if the system state is 0 , either the system will remain at state 0 should a home handoff takes place, or the system will go to state 1 should a local handoff takes place; if the system state is $d$, not mentioning the home handoff, the system's next state will be either $d+1$ or $d-1$ depending on whether the mobile node moves away from or close to its mailbox. We use a thick line to represent an action with $100 \%$ certainty and a thin line to represent an action with possibility. With all the necessary items for the dynamic programming defined, the backward induction algorithm can be applied to derive the optimal decision policy for the mailbox's migration. Readers are referred to [5] for details.

\section{Performance Evaluation}

In this section, we will evaluate the performance of our scheme with the adaptive algorithm. First, let us conduct the performance modeling for the benchmark scheme - Mobile IP route optimization with the smooth handoff extension. Fig. 8 depicts the network scenario about the mobile node's migration in this scheme. Here $d_{8}$ is the average distance from the Previous FA to all the correspondent nodes.

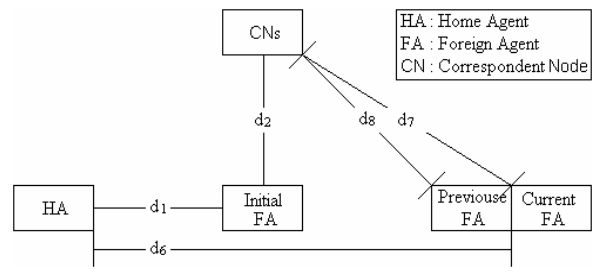

Fig. 8. Network scenario in the benchmark scheme

During each mobile node's migration, different types of signaling messages will be issued to the following entities: the home agent, the Previous FA and all the correspondent nodes. Therefore, Cost $_{\text {signaling }}(i)$ can be expressed as follows.

$$
\begin{gathered}
\operatorname{Cost}_{\text {signaling }}(\mathrm{i})=\left(\mathrm{w}+\mathrm{d}_{6}+1\right) \mathrm{d}_{\mathrm{Cs}}+\operatorname{Cost}_{\text {update }}(\mathrm{i}) \\
\operatorname{Cost}_{\text {update }}(\mathrm{i})=\mathrm{M} \times \mathrm{d}_{8} \times \mathrm{d}_{\mathrm{Cs}}
\end{gathered}
$$

To derive Cost $_{\text {packet }}(i)$, we have to separately deal with the normal packet delivery and the packet retransmission. Below list the expressions of the cost and the time period of the normal packet delivery, and those of the packet retransmission. The 
packet retransmission period is after the mobile node's migration to the new cell but before the "Previous Foreign Agent Notification" message arrives at the Previous FA.

$$
\begin{gathered}
\operatorname{Cost}_{\text {normal }}(\mathrm{i})=\left(\mathrm{d}_{7}+\mathrm{w}\right) \mathrm{d}_{\mathrm{Cp}} \\
\mathrm{T}_{\text {normal }}(\mathrm{i})=1 / \mu-\mathrm{T}_{\text {retransmission }}(\mathrm{i}) \\
\text { Cost }_{\text {retransmission }}(\mathrm{i})=\left(\mathrm{d}_{8}+\mathrm{w}+\mathrm{d}_{7}+\mathrm{w}\right) \mathrm{d}_{\mathrm{Cp}} \\
\mathrm{T}_{\text {retransmission }}(\mathrm{i})=(\mathrm{w}+1) \mathrm{d}_{\mathrm{Ts}}
\end{gathered}
$$

Therefore, Cost $_{\text {packet }}(i)$ can be expressed as follows.

$$
\begin{aligned}
\operatorname{Cost}_{\text {packet }}(\mathrm{i})= & \lambda \times \mathrm{T}_{\text {normal }}(\mathrm{i}) \times \operatorname{Cost}_{\text {normal }}(\mathrm{i})+ \\
& \lambda \times \mathrm{T}_{\text {retransmision }}(\mathrm{i}) \times \operatorname{Cost}_{\text {retransmision }}(\mathrm{i})
\end{aligned}
$$

As mentioned, our experiments start with the mobile node and its mailbox collocating at an initial foreign agent, where the distance to the home agent and the average distance to the corresponding nodes are $d_{1}$ and $d_{2}$, respectively. We selectively choose two $\left(d_{1} ; d_{2}\right)$ pairs: $(100 ; 0)$ and $(0 ; 100)$, where the first pair visualizes the scenario when the mobile node is far away from the home agent but close to its correspondent nodes, and the second pair shows the scenario in a reverse condition.

The experimental period lasts for 36 mobile node's migrations; the signaling transmission cost per hop $\mathrm{d}_{\mathrm{Cs}}$ is set to a normalized value 1; the packet delivery cost per hop $\mathrm{d}_{\mathrm{Cp}}$ is twice as high as $\mathrm{d}_{\mathrm{Cs}} ; \mathrm{d}_{\mathrm{Ts}}$, which can be understood as the signaling processing time on a router, is set to $0.05 \mathrm{sec}$; we assume the transmission cost (the transmission delay) in the wireless environment is twice as high (long) as that in the wired environment; and there are five active correspondent nodes.

Other parameters that affect the performance metric are $d_{4}, d_{6}, d_{7}, d_{8}, \eta$ and $K$. As the mobile node can move in any direction, the average value for $d_{6}$ should be the same as $d_{1}$ and the average values for $d_{4}, d_{7}$ and $d_{8}$ should be the same as $d_{2}$. For simplicity, we just replace $d_{6}$ with $d_{1}$, and $d_{4}, d_{7}$ and $d_{8}$ with $d_{2}$ in the equations. All these distance values may be obtained from the routing table if it uses link state routing protocols such as OSPF, and the packet-to-mobility ratio $\eta$ is easy to obtain since the mailbox acts as a relay and buffer station of the mobile node. We also select three $K$ s: 1,5 and 10 , where $K=1$ only considers the immediate benefit and $K=10$ takes more future influence into the consideration.

Fig. 9 lists the experimental results. For all six diagrams, we use $\eta$ as the $\mathrm{x}$-axis, and exam separately the signaling transmission cost, the packet forwarding cost and the total communication cost under different experimental settings. We observe that the signaling transmission cost increases as $\eta$ rises. This is because as more packets are received during each migration, it is more likely that the mailbox also moves, i.e., a home handoff occurs. For a home handoff, more signaling messages for the location registration are needed than a local handoff. However, the packet forwarding cost drops since after the mailbox's migration, packets are routed in a direct path (sender $\rightarrow$ receiver (mailbox)), instead of the triangle routing (sender $\rightarrow$ mailbox $\rightarrow$ receiver), between the sender and the receiver. 


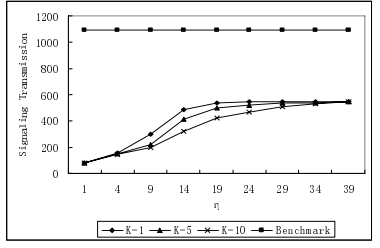

(a)

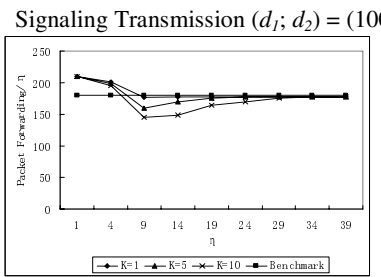

(c)

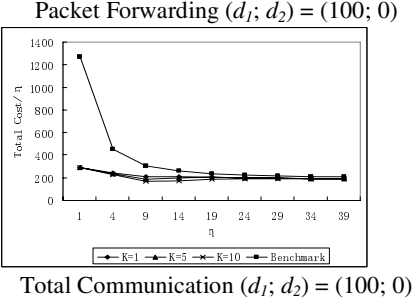

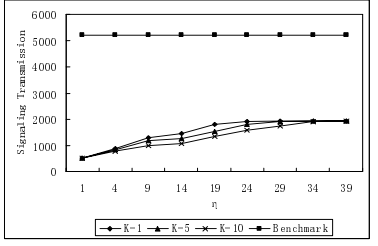

(b) Signaling Transmission $\left(d_{l} ; d_{2}\right)=(0 ; 100)$

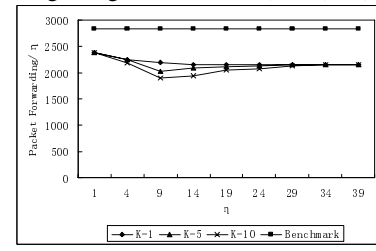

(d) Packet Forwarding $\left(d_{l} ; d_{2}\right)=(0 ; 100)$

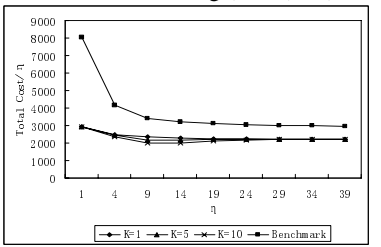

(f) Total Communication $\left(d_{l} ; d_{2}\right)=(0 ; 100)$

Fig. 9. Experimental result

We are pleased to discover that both the signaling transmission cost and the packet forwarding cost of our scheme are smaller than those of the smooth handoff scheme. For the signaling transmission cost, it is easy to understand the advantage of our scheme since our scheme normally does not require the home agent be notified of every mobile node's migration while the smooth handoff scheme does. For the packet forwarding cost, although the smooth handoff scheme may spend less for the normal packet transmission, it would require significant cost for retransmitting lost packets, especially when the distance to the senders $d_{2}$ is long. This is because in our scheme, the retransmission starts from the nearby mailbox while in the smooth handoff scheme, the retransmission starts from the possibly distant senders. Finally, the total cost of our scheme is smaller than that of the smooth handoff scheme.

Besides, a smaller $K$ normally performs worse than a bigger $K$, which implies that the dynamic programming configured with a smaller $K$ does not produce the optimal mailbox's migration policy. This is because a smaller $K$ only considers the myopic benefit without thinking about the future effect. Generally speaking, the bigger $K$ is, the more future effect is considered, the more optimal migration policy of the mailbox can be derived, and the less total communication cost is required.

\section{Conclusion}

In this paper, we propose a mailbox-based scheme with an adaptive algorithm to improve the performance of Mobile IP in the following aspects: reduced workload on 
the home agent, fast handoff, reduced packet loss, high throughput, reduced retransmission cost and delay, per-user-based adaptive location management, and dynamic tradeoff between the packet delivery cost and the location registration cost. The experiments conducted show a very sound result that demonstrates the benefits of using the mailbox, especially when the home agent is far away from the current location of the mobile node.

\section{Acknowledgment}

This work was supported by the National Natural Science Foundation of China under Grant No. 60673123 and the National Hi-Tech Research and Development 863 Program of China under Grant No. 2006AA01Z231.

\section{References}

1. Perkins, C.: IP Mobility Support for IPv4, RFC 3220 (January 2002)

2. Postel, J.: Multi-LAN Address Resolution, RFC 925 (October 1984)

3. Wang, Y., Chen, W., Ho, J.: Performance Analysis of Mobile IP Extended with Routing Agents. In: Proceedings of European IASTED International Conference on Parallel and Distributed Systems (July 1998)

4. Akyildiz, F., Ho, J., Lin, Y.: Movement-Based Location Update and Selective Paging for PCS Networks. The IEEE/ACM Transactions on Networking 4(4), 629-638 (1996)

5. Bellman, R.: Dynamic Programming. Princeton University Press, Princeton (1957)

6. Zhang, L., Cao, J., Das, S.K.: A Mailbox-based Scheme for Improving Mobile IP Performance. In: Proceedings of Mobile and Wireless Networks (May 2003) 